\title{
microRNA-544 promoted human osteosarcoma cell proliferation by downregulating AXIN2 expression
}

\author{
MING CHEN, YONG-YI LIU, MIN-QING ZHENG，XIN-LIANG WANG, \\ XING-HUA GAO, LIN CHEN and GUANG-MING ZHANG \\ Department of Orthopedics, Guangzhou First People's Hospital, \\ Guangzhou Medical University, Guangzhou, Guangdong 510180, P.R. China
}

Received July 13, 2016; Accepted January 12, 2018

DOI: $10.3892 / \mathrm{ol} .2018 .8218$

\begin{abstract}
RNAs (miRNAs) perform various oncogenic or tumor suppressor functions in carcinogenesis. Currently, the underlying mechanisms of miRNAs in osteosarcoma (OS) are poorly understood. In the present study, it is demonstrated that expression of miR-544 was markedly upregulated in OS cells and clinical tissues. Furthermore, overexpression of miR-544 enhanced OS cell proliferation in vitro. Bioinformatics analysis indicated that miR-544 may target the 3'-untranslated region of axis formation inhibitor 2 , which was validated using luciferase reporter gene assays. The present study demonstrated a vital role for miR-544 in promoting OS cell proliferation, indicating that it may represent a novel prognostic factor or therapeutic target for OS.
\end{abstract}

\section{Introduction}

Osteosarcoma (OS) is the most common type of primary malignant bone tumor diagnosed in children and adolescents (1). Despite advancements in multi-agent chemotherapies and surgical techniques, the prognosis for patients with OS remains poor, owing to its high recurrence rate and metastatic potential $(2,3)$. The precise mechanisms underlying OS carcinogenesis and progression remain largely unknown. Therefore, the elucidation of the mechanisms that mediate the initiation and progression of OS carcinogenesis, and an exploration of potential therapeutic agents are urgently required (4).

MicroRNAs (miRNAs) are a class of small non-coding regulatory RNAs (19-25 nucleotides) that serve roles essential for diverse biological processes, including cellular proliferation, migration, invasion and apoptosis (5-8). miRNAs usually

Correspondence to: Dr Guang-Ming Zhang, Department of Orthopedics, Guangzhou First People's Hospital, Guangzhou Medical University, 1 Panfu Road, Yiuxiu, Guangzhou, Guangdong 510180, P.R. China

E-mail: gmzhang2011@sina.com

Key words: microRNA-544, osteosarcoma, axis formation inhibitor 2, cell proliferation bind imperfectly to the 3'-untranslated regions (3'-UTRs) of target mRNAs. Previous studies have demonstrated that miR-544 serves pivotal roles in various types of cancer. miR-544 was reported to suppress proliferation, invasion and migration, and to induce cell apoptosis, in glioma by targeting Parkinsonism-associated deglycase (9). miR-544 was demonstrated to act as an oncogene in gastric cancer by repressing Iroquois homeobox 1 (10). However, to the best of our knowledge, the role of miR-544 in OS has not yet been reported. In the present study, we detected miR-544 expression in human OS tissues and cell lines, the biological effects and potential mechanisms of miR-544 in OS were investigated.

\section{Materials and methods}

Cell culture. Human OS cell lines, U2-OS, SAOS-2, MG-63 and SOSP-9607, and the human osteoblast cell line h-FOB, were purchased from the Cell Bank of Chinese Academy of Sciences (Shanghai, China) and grown in Dulbecco's modified Eagle's medium (DMEM; Gibco; Thermo Fisher Scientific, Inc., Waltham, MA, USA) supplemented with $10 \%$ fetal bovine serum (FBS; Sigma-Aldrich; Merck KGaA, Darmstadt, Germany) and $100 \mathrm{U} / \mathrm{ml}$ penicillin/streptomycin (Invitrogen; Thermo Fisher Scientific, Inc.). Cell lines were cultured in a humidified incubator at $37^{\circ} \mathrm{C}$ in an atmosphere containing $5 \% \mathrm{CO}_{2}$.

Clinical specimens. A total of 8 pairs of human OS tissues and tumor-adjacent normal tissues (TATs) were obtained from 8 patients (4 females and 4 males; age range, 31-55) with OS at the Department of Orthopedics, Guangzhou First People's Hospital (Guangzhou, China) from August 2014 to October 2015. The present study was approved by the Ethics Committee of Guangzhou First People's Hospital (Guangzhou, China). OS diagnosis was confirmed pathologically by 2 pathologists independently. Informed consent was obtained from all patients for tissue collection during surgery. Tissues were immediately frozen in liquid nitrogen, and stored at $-80^{\circ} \mathrm{C}$ until use.

Plasmids, small interfering (si)RNA and transfection. miR-544 mimics (HmiR0234), miR-544 inhibitor (miR-544-in, HmiR-AN0623), and the negative control 
miRNAs (CmiR0001 and CmiR-AN0001) were purchased from GeneCopoeia, Inc., (Rockville, MD, USA), and each miRNA (30 nM) was transfected into OS cells using Lipofectamine ${ }^{\circledR} 2000$ reagent (Invitrogen; Thermo Fisher Scientific, Inc.), according to the manufacturer's protocol. The time interval between transfection and subsequent experimentation was $48 \mathrm{~h}$.

AXIN2-siRNA (5'-GCAGAGGGACAGGAATCAT-3') and the negative control siRNA (5'-GCAGGGACAAGGTAG ACAT-3') were purchased from Qiagen, Inc., (Valencia, CA, USA). Transfection with $50 \mathrm{nM}$ siRNA was performed using Lipofectamine ${ }^{\circledR} 2000$ reagent, according to the manufacturer's protocol. The time interval between transfection and subsequent experimentation was $48 \mathrm{~h}$.

RNA extraction and reverse transcription-quantitative polymerase chain reaction $(R T-q P C R)$. Total RNA, including miRNA, was extracted from OS cells and clinical tissues using TRIzol ${ }^{\circledR}$ (Life Technologies; Thermo Fisher Scientific, Inc.), according to the manufacturer's protocol. miRNA was converted to cDNA using a TaqMan ${ }^{\circledR}$ miRNA Reverse Transcription kit (Applied Biosystems; Thermo Fisher Scientific, Inc.). The expression levels of miR-544 (HmiRQP0623, GeneCopoeia ${ }^{\mathrm{TM}}$, Guangzhou, China) were quantified using primers within a miRNA-specific TaqMan ${ }^{\circledR}$ miRNA assay kit (Applied Biosystems; Thermo Fisher Scientific, Inc), according to the manufacturer's protocol. PCR was performed to detect the expression of Cyclin D1 (CCND1, HQP016204, GeneCopoeia ${ }^{\mathrm{TM}}$, Guangzhou, China) and c-Myc (HQP011597, GeneCopoeia ${ }^{\mathrm{TM}}$, Guangzhou, China using the ABI 7500 Fast Real-Time PCR system (Thermo Fisher Scientific, Inc.) with SYBR Green Mix Taq kit (Takara Bio, Inc., Otsu, Shiga, Japan). The thermocycling conditions were as follows: At $95^{\circ} \mathrm{C}$ for $30 \mathrm{sec}$, followed by 40 cycles of amplification at $95^{\circ} \mathrm{C}$ for $5 \mathrm{sec}$, at $59^{\circ} \mathrm{C}$ for $30 \mathrm{sec}$ and at $72^{\circ} \mathrm{C}$ for 30 sec. U6 (HmiRQP9001, GeneCopoeia ${ }^{\mathrm{TM}}$, Guangzhou, China) and GAPDH (HQP064347, GeneCopoeia ${ }^{\mathrm{TM}}$, Guangzhou, China) served as internal controls for the miRNA and mRNA assays, respectively. Expression was quantified using the $2^{-\Delta \Delta \mathrm{Cq}}$ method (11).

MTT assay. Transfected SOSP-9607 cells were seeded into 96-well plates, in medium containing $10 \%$ FBS, at a density of $1 \times 10^{3}$ cells/well. The cells were stained with $20 \mu \mathrm{g}$ MTT dye (0.5 mg/ml; Sigma-Aldrich; Merck KGaA). The formazan crystals formed were dissolved in $150 \mu \mathrm{l}$ dimethyl sulfoxide (DMSO; Sigma-Aldrich; Merck KGaA) at 1, 2, 3, 4 or 5 days, and the absorbance was recorded at $490 \mathrm{~nm}$ using a spectro-photometric plate reader.

Colony formation assay. SOSP-9607 cells were seeded into 6-well plates $\left(1 \times 10^{3}\right.$ cells/well) and incubated for 10 days in medium containing $10 \% \mathrm{FBS}$. The colonies were stained with $0.5 \%$ crystal violet at room temperature for $15 \mathrm{~min}$ following fixation in $4 \%$ paraformaldehyde for $5 \mathrm{~min}$ at room temperature. The number of colonies, each defined as a group of $>50$ cells, was counted per plate.

Anchorage-independent growth assay. SOSP-9607 cells were trypsinized, and $2 \times 10^{3}$ cells were suspended in complete medium (Dulbecco's modified Eagle's medium supplemented with $10 \% \mathrm{FBS}$ and $100 \mathrm{U} / \mathrm{ml}$ penicillin/streptomycin) containing $0.3 \%$ agar (Sigma-Aldrich; Merck KGaA), and applied onto a layer of $1 \%$ agar in complete medium in 6-well plates. Cells were incubated for 2 weeks at $37^{\circ} \mathrm{C}$ prior to subjection to a colony formation assay, as aforementioned, and cell colonies were imaged at magnification x100. Only cell colonies $>0.1 \mathrm{~mm}$ in diameter were counted.

MiRNA target prediction and Dual-luciferase reporter assay. Based on the miR sequences, target genes were predicted using TargetScan (version 3.1; http://www.targetscan. org/mamm_31/). AXIN2 was amplified from SOSP-9607 cell cDNA and was sub-cloned into a firefly luciferase reporter pGL3 plasmid (cat. no., GUR100013-P-2; Guangzhou RiboBio Co., Ltd., Guangzhou, China). Cells were seeded in triplicate in 24 -well plates $\left(5 \times 10^{4}\right.$ cells/well) and cultured for 24 h. pGL3-AXIN2-3L3-luciferase reporter pGL3 plasmids were co-transfected into the cells with the pRL-TK Renilla plasmids (Promega Corporation, Madison, WI, USA) using Lipofectamine $^{\circledR} 2000$ (Invitrogen; Thermo Fisher Scientific, Inc.), according to the manufacturer's protocol. After $48 \mathrm{~h}$ of transfection, the luciferase activities of the transformed cells were assayed using a Dual-Luciferase Reporter assay system (Promega Corporation), according to the manufacturer's protocol. Firefly luciferase activity was normalized to Renilla luciferase activity.

Western blotting. Total protein was extracted using radioimmunoprecipitation assay buffer (Beyotime Institute of Biotechnology, Haimen, China), and protein concentrations were determined with BCA Protein Assay Kit (Beyotime, Institute of Biotechnology), accoding to the manufacturer's protocol. A total of $50 \mu \mathrm{g}$ protein extracts were separated via $10 \%$ SDS-PAGE and transferred onto PVDF membranes. The membranes were blocked with $5 \%$ non-fat milk in TBS-T (20 mM Tris, pH 7.6, $137 \mathrm{mM} \mathrm{NaCl,} \mathrm{0.05 \%} \mathrm{Tween-20)} \mathrm{for}$ $0.5 \mathrm{~h}$ at room temperature, and then incubated overnight with anti-AXIN2 (catalog no., 2151), anti-CCND1 (catalog no., 2978) and anti-c-Myc (catalog no., 13987) antibodies (all at a dilution of 1:1,000; Cell Signaling Technology Inc., Danvers, MA, USA) at $4^{\circ} \mathrm{C}$ overnight. $\alpha$-tubulin (catalog no., 2144; 1:5,000; Sigma-Aldrich; Merck KGaA) was used as the loading control. The blots were then incubated for $2 \mathrm{~h}$ with a horseradish peroxidase-conjugated anti-rabbit immunoglobulin secondary antibody (cat no. P0023D; 1:5,000; Beyotime Institute of Biotechnology) at $37^{\circ} \mathrm{C}$. Signals were visualized using enhanced chemiluminescence (Thermo Fisher Scientific, Inc.) as a substrate, and images were analyzed using an automated chemiluminescence system (LAS500; GE Healthcare, USA) according to protocol of the manufacturer.

Statistical analysis. All data are presented as the mean \pm standard deviation, and all experiments were repeated independently at least 3 times. Statistical analyses, specifically a one-way analysis of variance (ANOVA) or the Student's t test, were performed using SPSS 17.0 software (SPSS Inc., Chicago, IL, USA). $\mathrm{P}<0.05$ was considered to indicate a statistically significant difference. 
A

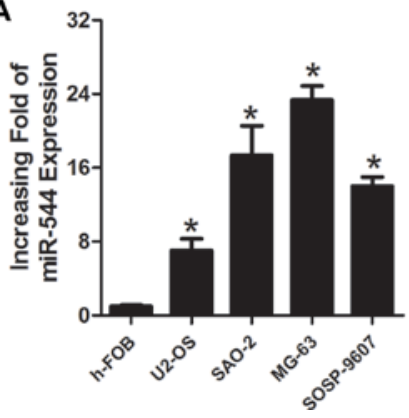

B

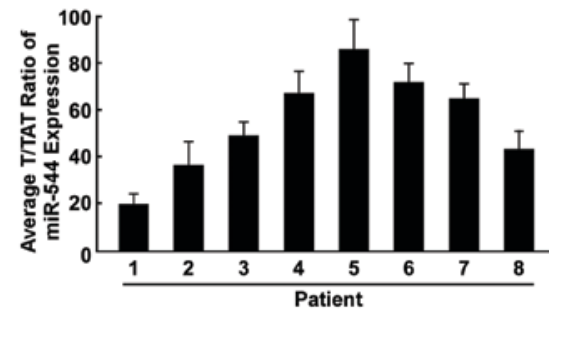

Figure 1. Expression of miR-544 in human OS cell lines and tissues. (A) RT-qPCR analysis of miR-544 expression in human osteoblast h-FOB cells, and in the OS cell lines U2-OS, SAO-2, MG-63 and SOSP-9607. "P<0.05 vs. h-FOB. (B) Relative miR-544 expression levels in eight paired primary Ts and TATs from 8 patients were detected by RT-qPCR. Each bar represents the mean of 3 independent experiments, and error bars represent the standard deviation. miR, microRNA; OS, osteosarcoma; RT-qPCR, reverse transcription-quantitative polymerase chain reaction; T, OS tissue; TAT, tumor-adjacent normal tissues.

A

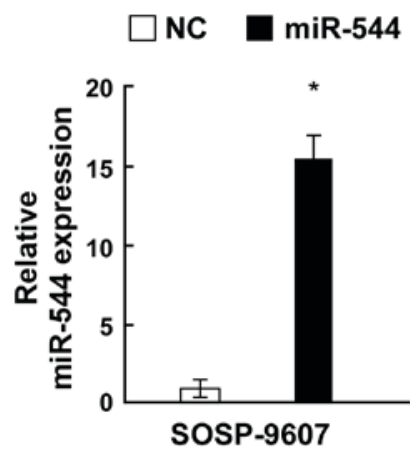

C

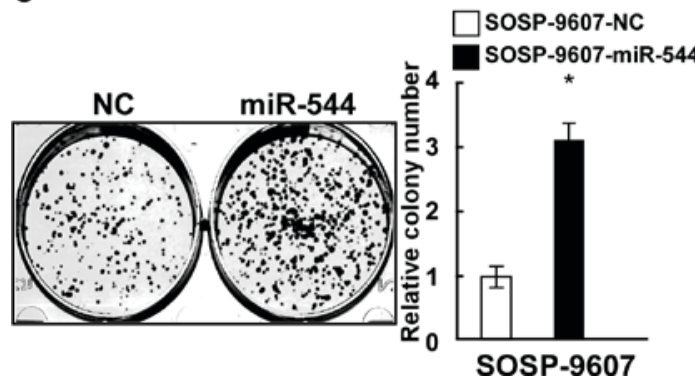

B

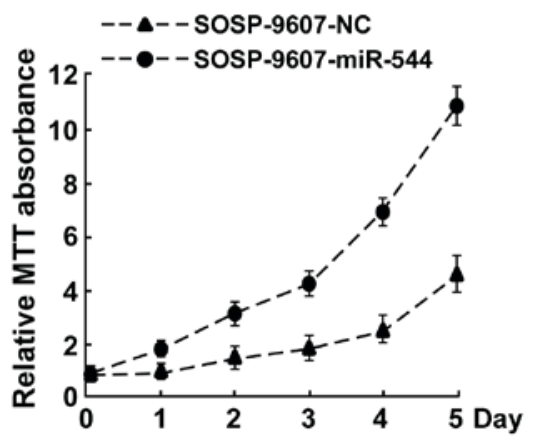

D

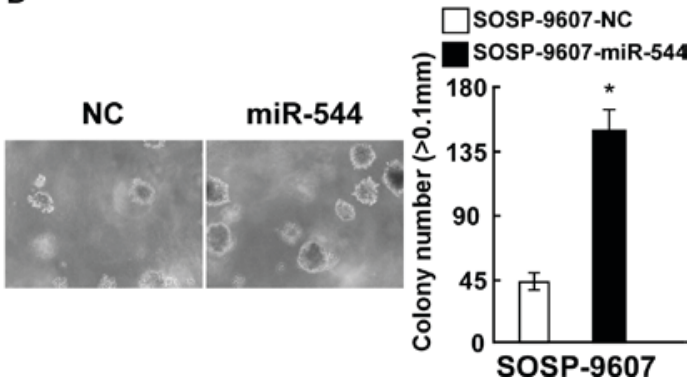

Figure 2. miR-544 upregulation promotes OS cell proliferation. (A) RT-qPCR analysis validation of increased miR-544 expression after transfection with miR-544 mimics. (B) MTT assays showed that the result of relative MTT absorbance (relative to the results at day 0 ) revealed that miR-544 promoted the growth of SOSP-9607 OS cells. (C) Quantification of crystal violet-stained cell colonies, relative to those cells transfected with the NC. (D) Upregulation of miR-544 promoted the anchorage-independent growth of SOSP-9607 cells. Representative micrographs (left, x200 magnification) and quantification of colony numbers (right). Each bar represents the mean of 3 independent experiments, and the error bars represent the standard deviation. "P<0.05, compared with cells transfected with the NC. miR, microRNA; OS, osteosarcoma; RT-qPCR, reverse transcription-quantitative polymerase chain reaction; NC, negative control.

\section{Results}

miR-544 expression is upregulated in OS cell lines and tissues. To investigate the role of miR-544 in OS development, miR-544 expression was examined in OS cells and clinical tissue samples. RT-qPCR analysis indicated that miR-544 expression was significantly increased in the OS cell lines, U2-OS, SAO-2, MG-63 and SOSP-9607, compared with in the human osteoblast h-FOB cells (Fig. 1A). Furthermore, miR-544 expression was markedly upregulated in the OS tissues, as compared with in the matched TATs (Fig. 1B). This suggests that miR-544 is upregulated in OS and that it may serve a role in promoting OS development.
miR-544 promotes $O S$ cell proliferation and cell cycle progression. To explore the effect of miR-544 on OS cell proliferation, SOSP-9607 cells were transfected with miR-544 mimics, a miR-544-in or the respective controls, and the expression of miR-544 in the stably transfected SOSP-9607 cell line was confirmed by RT-qPCR (Fig. 2A). MTT and colony formation assays revealed that the overexpression of miR-544 increased the proliferation of SOSP-9607 cells, as compared with those cells transfected with negative control miRNA (Fig. 2B and C). Overexpression of miR-544 in SOSP-9607 cells also significantly enhanced their anchorage-independent growth ability $(\mathrm{P}<0.05$; Fig. 2D). By contrast, SOSP-9607 cells were transfected with miR-544-in or the respective 
A

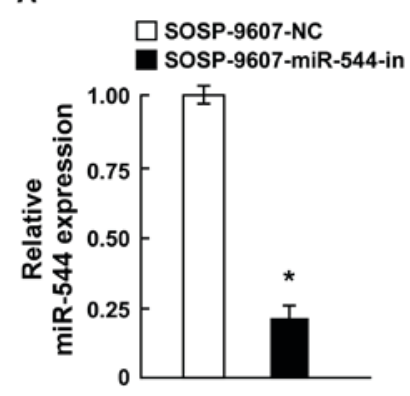

C

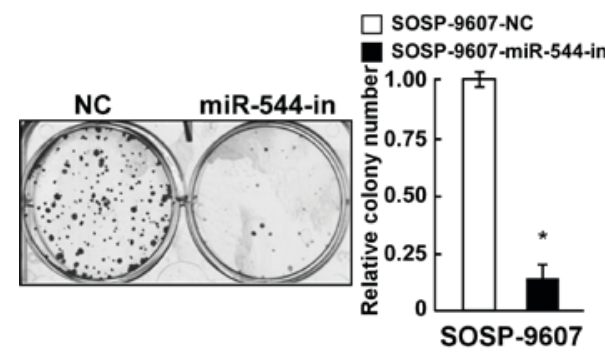

B

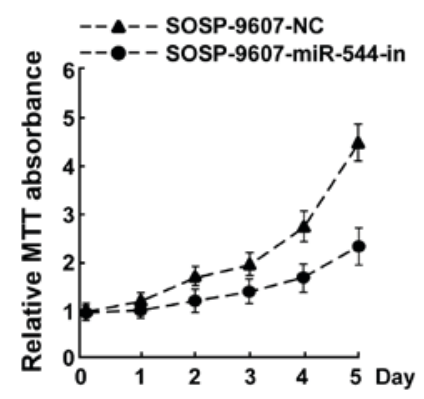

D

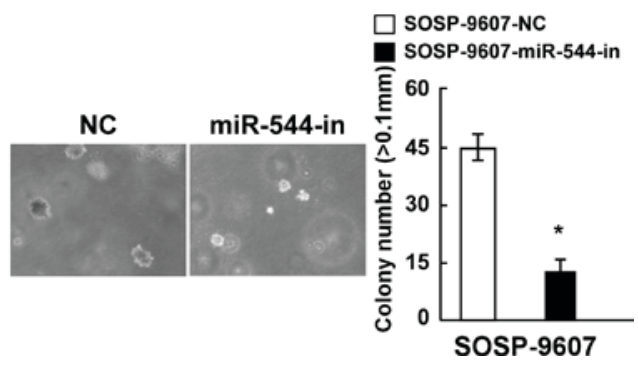

Figure 3. Inhibition of miR-544 reduced OS cell proliferation. (A) Validation of miR-544 expression levels following transfection with miR-544-in by RT-qPCR analysis. (B) MTT assay showed that the result of relative MTT absorbance (relative to the results of each group at day 0 ) revealed that the downregulation of miR-544 inhibited the growth of SOSP-9607 cells. (C) Quantification of crystal violet-stained cell colonies, relative to cells transfected with the NC. (D) Inhibition of miR-544 decreased the anchorage-independent growth of SOSP-9607 cells. Representative micrographs (left, x200, magnification) and quantification of colony number (right). Each bar represents the mean of 3 independent experiments, and the error bars represent the standard deviation. ${ }^{*} \mathrm{P}<0.05$, compared with cells transfected with the NC. miR, microRNA; OS, osteosarcoma; RT-qPCR, reverse transcription-quantitative polymerase chain reaction; $\mathrm{NC}$, negative control.

control, and the expression of miR-544 in the stably transfected SOSP-9607 cell line was confirmed by RT-qPCR (Fig. 3A). MiR544-in-transfected SOSP-9607 cells exhibited decreased proliferation rates, colony formation ability, and anchorage-independent cell growth ability, compared with in the negative control cells (Fig. 3B-D). Collectively, these data indicate that miR-544 acted as a tumor promoter to endorse OS cell proliferation.

miR-544 directly targets AXIN2 by binding its 3'-UTR in OS and alters the expression of proliferation-associated proteins. According to bioinformatical predictions, AXIN2 is a putative target gene of miR-544. To verify this prediction, WT AXIN2 3'UTRs were generated (Fig. 4A). To determine whether miR-544 affected AXIN2 expression, AXIN2 expression was analyzed by western blotting. Overexpression of miR-544 inhibited the protein expression of AXIN2, while SOSP-9607 cells transfected with miR-544-in exhibited enhanced protein expression of AXIN2 (Fig. 4B). To investigate whether AXIN2 could be regulated by miR-544, AXIN2 3'-UTR was co-transfected into SOSP-9607 cells with miR-544 mimics, miR-544-in or miR-544-mut, followed by the measurement of luciferase activity. As demonstrated in Fig. 4C, luciferase activity was markedly reduced in cells that were co-transfected with the WT AXIN2 3'-UTR and miR-544. By contrast, miR-544-in transfection increased luciferase activity in cells transfected with WT AXIN2 3'-UTR. miR-544-mut transfection did not alter the luciferase activity of cells transfected with AXIN2 3'-UTR. These findings indicate that miR-544 downregulated the protein expression of AXIN2 via direct binding to the seed sequences in its 3'-UTR.
It has been reported that the Wnt signaling pathway serves an essential role in the cancer cell cycle and proliferation $(12,13)$. C-Myc, CCND1, and AXIN2 are well-known target genes of the Wnt signaling pathway (14-17). The expression of these Wnt/ $\beta$-catenin signaling pathway downstream genes was determined in miR-544-transfected cells. Using RT-qPCR and western blotting, it was demonstrated that the mRNA and protein expression levels of CCND1 and c-Myc were upregulated in miR-544-transfected SOSP-9607 cells, while transfection with miR-544-in resulted in the opposite effect (Fig. 4D and E). This suggests that miR-544 modulated downstream genes of the Wnt signaling pathway (AXIN2, CCND1 and c-Myc).

AXIN2 suppression counteracts the proliferation arrest induced by miR-544-in. The effects of AXIN2 downregulation on proliferation were examined in OS cells transfected with miR-544-in. siRNA-mediated knockdown of AXIN2 was performed in miR-544-in-transfected SOSP-9607 cells, and confirmed through western blotting (Fig. 5A). Results from the colony formation and anchorage-independent growth assays indicated that the suppression of AXIN2 expression by AXIN2-siRNA reversed the effects of miR-544-in in SOSP-9607 cells (Fig. 5B and C). Taken together, these results demonstrated that the downregulation of AXIN2 counteracts the cell proliferation arrest induced by miR-544-in.

\section{Discussion}

Prior studies have demonstrated that microRNAs negatively regulate their target mRNAs in a sequence-specific manner $(18,19)$, to serve roles in the development of human 
A

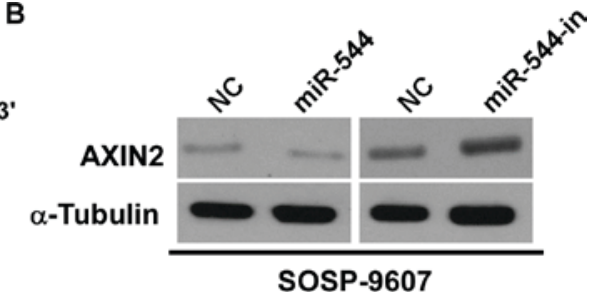

D

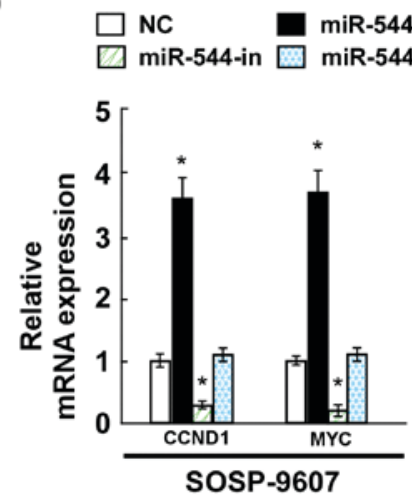

E

C

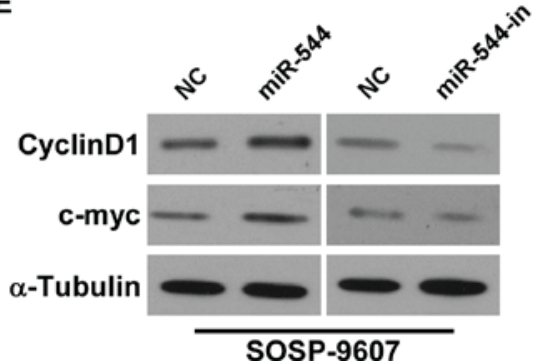

SOSP-9607

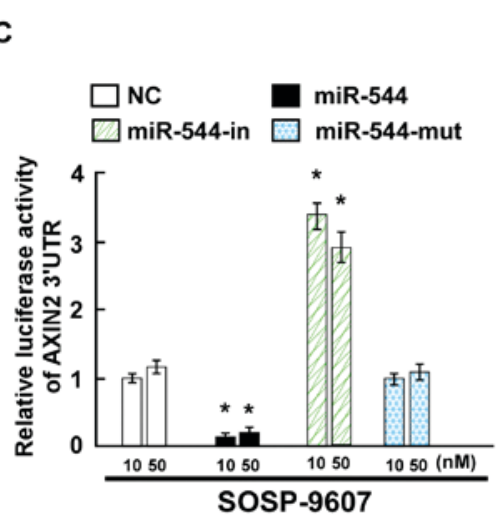

miR-544 5' 395 CCUACCAUUGACACAUGCAGAAA 608
3' CUUGAACGAUUUUUACGUCUUA 5'

miR-544-mut 3' CUUGAACGAUUUUUACGCAGUA 5'

Figure 4. miR-544 suppresses AXIN2 expression by directly targeting the AXIN2 3'-UTR, and alters the levels of proteins associated with proliferation in SOSP-9607 cells. (A) Predicted miR-544 target sequence in the three cells ng AXIN2 (AXIN2-3'-UTR) and positions of three mutated nucleotides (green) in the 3'-UTR of miR-544 mut. (B) Western blotting analysis of AXIN2 expression in cells transfected with miR-544 or the miR-544-in. $\alpha$-tubulin served as the loading control. (C) Luciferase reporter assay of SOSP-9607 cells transfected with the pGL3-AXIN2-3'-UTR and miR-544 or miR-544-in or miR-544-mut siRNA. "P<0.05 vs. NC. (D) Reverse transcription-quantitative polymerase chain reaction analysis of the expression of CCND1 and c-Myc in the indicated SOSP-9607 cells. vs. NC "P<0.05 (E) Western blot analysis of the expression of CCND1 and c-Myc in SOSP-9607 cells. $\alpha$-tubulin served as the loading control. miR, microRNA; AXIN2, axis formation inhibitor 2; UTR, untranslated region; CCND1, cyclin D1; NC, negative control; -in, inhibitor; mut, mutated; si, small interfering.

A

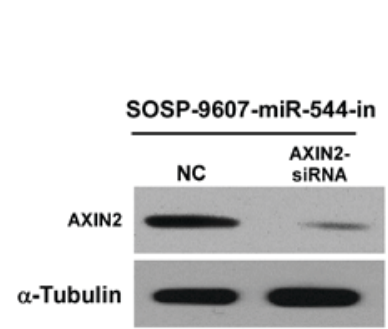

B

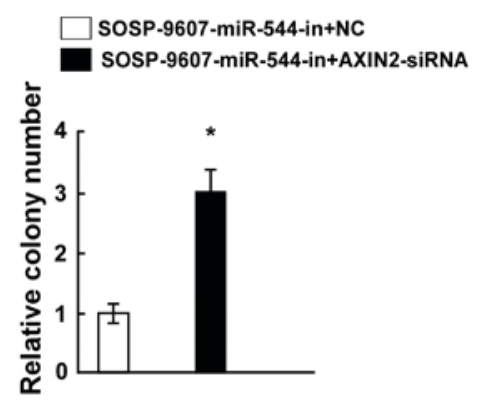

C

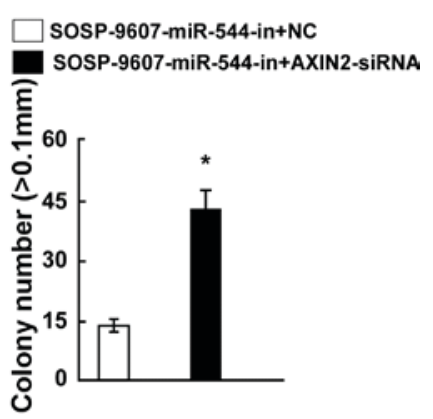

Figure 5. AXIN2 suppression counteracts the proliferation arrest induced by miR-544-in. (A) Western blot analysis verified that AXIN2 siRNA transfection effectively decreased the expression of AXIN2 in miR-544-in-transfected SOSP-9607 cells. (B) miR-544-in and AXIN2-siRNA-transfected SOSP-9607 cells exhibited increased colony formation, relative to the NC cells. (C) miR-544-in and AXIN2-siRNA-transfected SOSP-9607 cells exhibited increased anchorage-independent growth, relative to the NC. "P $<0.05$, compared with the NC. miR, microRNA; AXIN2, axis formation inhibitor 2; NC, negative control, -in, inhibitor; si, small interfering.

cancer types, including OS. miR-143 was reported to promote the apoptosis of OS cells by targeting Bcl-2 (20). miR-99a was demonstrated to inhibit cell proliferation by targeting TNF- $\alpha$-induced protein 8 in OS cells (21). Zhang et al (22) 
indicated that miR-30a regulates the proliferation, migration and invasion of human OS by regulating Runt-related transcription factor 2. However, to the best of our knowledge, the role of miR-544 in OS has not been previously investigated. miR-544 was demonstrated to suppress tumor growth in human triple-negative breast cancer by downregulating both B-cell CLL/lymphoma 6 and signal transducer and activator of transcription 3 (23). In the present study, it was demonstrated that miR-544 serves a role in promoting OS cell proliferation. miR-544 expression was increased in OS cell lines and tissues, compared in with h-FOB cells and TATs. The overexpression of miR-544 significantly increased OS cell proliferation. By contrast, miR-544-in-transfection significantly decreased cell proliferative ability, suggesting that miR-544 may be a novel tumor promoter and serve a critical role in OS carcinogenesis.

The results of the present study indicate that AXIN2 was a direct target gene of miR-544, and that it was implicated in the functional effect of miR-544 on OS carcinogenesis. It has been reported that AXIN2 is an important regulator of the Wnt/ $\beta$-catenin signaling pathway, and participates in various cellular functions $(24,25)$. Wei et al (26) suggested that AXIN2 expression was downregulated in ameloblastoma, and was involved in its tumorigenesis. Koinuma et al (27) indicated that epigenetic silencing of AXIN2 was associated with colorectal cancer carcinogenesis. Growing evidence suggests that AXIN2 can act as a tumor suppressor gene or an oncogene, regulated by several miRNAs. miR-374a was reported to promote esophageal cancer cell proliferation by suppressing AXIN2 expression (28). Additionally, miR-107 was determined to promote hepatocellular carcinoma cell proliferation by regulating AXIN2 (29). Similarly, Kim et al (30) indicated that miR-205 inhibited oral carcinoma oncogenic activity by downregulating AXIN2 expression. In the present study, the expression of CCND1 and c-Myc was upregulated, AXIN2 expression was suppressed by miR-544, and cell proliferation was elevated in OS. Furthermore, the knockdown of AXIN2 in miR-544-in-transfected SOSP-9607 OS cells counteracted the proliferation arrest induced by miR-544-in.

In conclusion, the results of the present study demonstrated that miR-544 directly regulates AXIN2 expression and, thus, contributes to OS tumorigenesis. This provides a novel insight into the biology of OS, and suggests that miR-544 may be a promising prognostic factor and therapeutic target for future OS therapy.

\section{Acknowledgements}

Not applicable.

\section{Funding}

This work was supported by Guangdong Science and Technology Project (2017ZC0320) and the Department of Orthopedics of Guangzhou First People's Hospital (Guangzhou, China).

\section{Availability of data and materials}

All data generated or analyzed during this study are included in this published article.

\section{Author's contributions}

GMZ and MC conceived and designed the experiments. MC, YYL, MQZ and XLW performed the experiments. XHG and LC collected the samples and analyzed the data. MC wrote the paper. The final manuscript was also approved by all authors.

\section{Ethics approval and consent to participate}

The present study was approved by the Ethics Committee of Guangzhou First People's Hospital (Guangzhou, China), and informed consent was obtained from all patients for tissue collection during surgery.

\section{Consent for publication}

Not applicable.

\section{Competing interests}

The authors declare that they have no competing interests.

\section{References}

1. Yu W, Zhu J, Wang Y, Wang J, Fang W, Xia K, Shao J, Wu M, Liu B, Liang C, et al: A review and outlook in the treatment of osteosarcoma and other deep tumors with photodynamic therapy: From basic to deep. Oncotarget 8: 39833-39848, 2017.

2. Nouri H, Ben Maitigue M, Abid L, Nouri N, Abdelkader A, Bouaziz M and Mestiri M: Surface osteosarcoma: Clinical features and therapeutic implications. J Bone Oncol 4: 115-123, 2015.

3. Anderson ME: Update on survival in osteosarcoma. Orthop Clin North Am 47: 283-292, 2016

4. Miao J, Wu S, Peng Z, Tania M and Zhang C: MicroRNAs in osteosarcoma: Diagnostic and therapeutic aspects. Tumour Biol 34: 2093-2098, 2013.

5. Liang WL, Cao J, Xu B, Yang P, Shen F, Sun Z, Li WL, Wang Q and Liu F: miR-892a regulated PPP2R2A expression and promoted cell proliferation of human colorectal cancer cells. Biomed Pharmacother 72: 119-124, 2015.

6. Zhang C, Long F, Wan J, Hu Y and He H: MicroRNA-205 acts as a tumor suppressor in osteosarcoma via targeting RUNX2. Oncol Rep 35: 3275-3284, 2016.

7. Liu ZF, Liang ZQ, Li L, Zhou YB, Wang ZB, Gu WF, Tu LY and Zhao J: miR-335 functions as a tumor suppressor and regulates survivin expression in osteosarcoma. Eur Rev Med Pharmacol Sci 20: 1251-1257, 2016

8. Ma C, Zhan C, Yuan H, Cui Y and Zhang Z: MicroRNA-603 functions as an oncogene by suppressing BRCC2 protein translation in osteosarcoma. Oncol Rep 35: 3257-3264, 2016.

9. Jin S, Dai Y, Li C, Fang X, Han H and Wang D: MicroRNA-544 inhibits glioma proliferation, invasion and migration but induces cell apoptosis by targeting PARK7. Am J Transl Res 8: 1826-1837, 2016.

10. Zhi Q, Guo X, Guo L, Zhang R, Jiang J, Ji J, Zhang J, Zhang J, Chen X, Cai Q, et al: Oncogenic miR-544 is an important molecular target in gastric cancer. Anticancer Agents Med Chem 13: 270-275, 2013.

11. Livak KJ and Schmittgen TD: Analysis of relative gene expression data using real-time quantitative PCR and the 2(-Delta Delta C(T)) method. Methods 25: 402-408, 2001.

12. Dai G, Zheng D, Wang Q, Yang J, Liu G, Song Q, Sun X, Tao C, $\mathrm{Hu} \mathrm{Q}$, Gao T, et al: Baicalein inhibits progression of osteosarcoma cells through inactivation of the Wnt/ $\beta$-catenin signaling pathway. Oncotarget 8: 86098-86116, 2017.

13. Feng ZY, Xu XH, Cen DZ, Luo CY and Wu SB: miR-590-3p promotes colon cancer cell proliferation via $\mathrm{Wnt} / \beta$-catenin signaling pathway by inhibiting WIF1 and DKK1. Eur Rev Med Pharmacol Sci 21: 4844-4852, 2017.

14. Leung JY, Kolligs FT, Wu R, Zhai Y, Kuick R, Hanash S, Cho KR and Fearon ER: Activation of AXIN2 expression by beta-catenin-T cell factor. A feedback repressor pathway regulating Wnt signaling. J Biol Chem 277: 21657-21665, 2002. 
15. Peng Z, Wu T, Li Y, Xu Z, Zhang S, Liu B, Chen Q and Tian D: MicroRNA-370-3p inhibits human glioma cell proliferation and induces cell cycle arrest by directly targeting $\beta$-catenin. Brain Res 1644: 53-61, 2016.

16. Eo HJ, Park GH and Jeong JB: Inhibition of Wnt signaling by silymarin in human colorectal cancer cells. Biomol Ther (Seoul) 24: 380-386, 2016.

17. Zhang W, Shen C, Li C, Yang G, Liu H, Chen X, Zhu D, Zou H, Zhen Y, Zhang D and Zhao S: miR-577 inhibits glioblastoma tumor growth via the Wnt signaling pathway. Mol Carcinog 55: $575-585,2016$.

18. van Kouwenhove M, Kedde M and Agami R: MicroRNA regulation by RNA-binding proteins and its implications for cancer. Nat Rev Cancer 11: 644-656, 2011

19. Calin GA and Croce CM: MicroRNA signatures in human cancers. Nat Rev Cancer 6: 857-866, 2006.

20. Li WH, Wu HJ, Li YX, Pan HG, Meng T and Wang X: MicroRNA-143 promotes apoptosis of osteosarcoma cells by caspase-3 activation via targeting Bcl-2. Biomed Pharmacother 80: 8-15, 2016.

21. Xing B and Ren C: Tumor-suppressive miR-99a inhibits cell proliferation via targeting of TNFAIP8 in osteosarcoma cells. Am J Transl Res 8: 1082-1090, 2016.

22. Zhang R, Yan S, Wang J, Deng F, Guo Y, Li Y, Fan M, Song Q, Liu $\mathrm{H}$, Weng $\mathrm{Y}$ and Shi Q: miR-30a regulates the proliferation, migration, and invasion of humanosteosarcomabytargetingRunx2.TumourBiol37:3479-3488, 2016.

23. Zhu Z, Wang S, Zhu J, Yang Q, Dong $H$ and Huang J: MicroRNA-544 down-regulates both Bcl6 and Stat3 to inhibit tumor growth of human triple negative breast cancer. Biol Chem 397: 1087-1095, 2016.
24. Liu H, Mastriani E, Yan ZQ, Yin SY, Zeng Z, Wang H, Li QH, Liu HY, Wang X, Bao HX, et al: SOX7 co-regulates Wnt $/ \beta$-catenin signaling with Axin-2: Both expressed at low levels in breast cancer. Sci Rep 6: 26136, 2016.

25. Yanaka Y, Muramatsu T, Uetake H, Kozaki K and Inazawa J: miR-544a induces epithelial-mesenchymal transition through the activation of WNT signaling pathway in gastric cancer. Carcinogenesis 36: 1363-1371, 2015.

26. Wei Z, Zhong M, Guo Y, Wang Y, Ren M and Wang Z: Expression of $\beta$-catenin and AXIN2 in ameloblastomas. Contemp Oncol (Pozn) 17: 250-256, 2013.

27. Koinuma K, Yamashita Y, Liu W, Hatanaka H, Kurashina K, Wada T, Takada S, Kaneda R, Choi YL, Fujiwara SI, et al: Epigenetic silencing of AXIN2 in colorectal carcinoma with microsatellite instability. Oncogene 25: 139-146, 2006.

28. Wang Y, Xin H, Han Z, Sun H, Gao N and Yu H: MicroRNA-374a promotes esophageal cancer cell proliferation via Axin2 suppression. Oncol Rep 34: 1988-1994, 2015.

29. Zhang JJ, Wang CY, Hua L, Yao KH, Chen JT and Hu JH: miR-107 promotes hepatocellular carcinoma cell proliferation by targeting Axin2. Int J Clin Exp Pathol 8: 5168-5174, 2015.

30. Kim JS, Park SY, Lee SA, Park MG, Yu SK, Lee MH, Park MR, Kim SG, Oh JS, Lee SY, et al: MicroRNA-205 suppresses the oral carcinoma oncogenic activity via down-regulation of Axin-2 in KB human oral cancer cell. Mol Cell Biochem 387: 71-79, 2014.

(i) (3) This work is licensed under a Creative Commons Attribution-NonCommercial-NoDerivatives 4.0 International (CC BY-NC-ND 4.0) License. 